\title{
Muscle-Eye-Brain Disease; a Rare Form of Syndromic Congenital Muscular Dystrophy
}

\author{
Gosal Gurinder S, MS Orth, Shah Hitesh H, MS Orth DNB \\ Paediatric Orthopaedics Service, Kasturba Medical College, Karnataka, India
}

\begin{abstract}
Congenital muscular dystrophy (CMD) is a heterogeneous group of disorders characterized by muscular hypotonia since birth and the histologic features of muscular dystrophy. Syndromic congenital muscular dystrophies are clinically similar autosomal recessive disorders characterized by congenital muscular dystrophy, lissencephaly, and eye anomalies.
\end{abstract}

We present a case of a rare form of syndromic congenital muscular dystrophy in an eight year old girl, born of firstdegree consanguinity. She had: global developmental delay; a seizure disorder; hypotonia; progressive muscle contractures including bilateral symmetrical flexion contractures of hips, knees, equinus contracture and thoracolumbar scoliosis; diminished deep tendon reflexes: bilateral premature cataract; pseudophakia; and nystagmus. The patient was also highly myopic. Based on clinical features, muscle biopsy and MRI of the brain, a diagnosis of muscle- eye- brain disease was made. Identification of these patients may help to prevent this crippling disorder in the future siblings of probands by utilizing genetic counselling and mutation analysis.

\section{Key Words:}

Congenital Muscular Dystrophy, Muscle-eye-brain Disease, Walker-Warburg Syndrome, Fukuyama Congenital Muscular Dystrophy.

\section{INTRODUCTION}

The congenital muscular dystrophies (CMDs) are a group of genetically and clinically heterogeneous hereditary myopathies with preferentially autosomal recessive inheritance, characterized by congenital hypotonia, delayed motor development and early onset of progressive muscle weakness associated with a dystrophic pattern on muscle biopsy ${ }^{1}$. The genotype-phenotype correlations in syndromic muscular dystrophies with defective glycosylation of alphadystroglycans form a broad clinical spectrum ${ }^{1,2,3}$. Within this spectrum, Walker-Warburg syndrome (WWS), muscleeye-brain disease (MEB) and Fukuyama congenital muscular dystrophy (FCMD) comprise the most severe conditions and are associated with varying degrees of defective brain migration and eye abnormalities ${ }^{1,2,3}$.

The diagnosis is established on the basis of four criteria: 1) congenital muscular dystrophy characterized by hypoglycosylation of $\alpha$-dystroglycans; 2) high creatine kinase level; 3) anterior or posterior eye anomalies; and, 4) migration brain defect with type II lissencephaly.

The various types can be further distinguished with Immunohistochemistry and genetic testing. Among these, alpha-dystroglycanopathies, muscle-eye-brain disease is prevalent mainly in Finland ${ }^{1,2,3,4}$. We could not find any similar report from India.

\section{CASE REPORT}

An eight year old female presented with multiple joint contractures and motor predominant global developmental delay. She was born out of consanguineous marriage (Figure.1), at full term, via a normal delivery and had an uneventful perinatal period. She was apparently well till 5 months of age when parents noticed that the infant was "floppy". She attained head control at 18 months of age. She never sat or stood independently. At 3 years of age, she suffered febrile seizures and was prescribed antiepileptic medication for a period of 3 years. She started speaking 2-3 words at 3 years and full sentences at 5 years of age. Eventually she developed progressive muscle contractures involving the hip, knee and ankle joints bilaterally. In addition, she underwent bilateral cataract surgery at age of 6 years, as well as a brain MRI and muscle biopsy.

On clinical evaluation, she had right thoracolumbar scoliosis, bilateral 60 degrees flexion contractures of the hip joints, 90 degrees flexion deformity of the knee joints and equinus contracture at the ankles (Figure 2) without any pseudohypertrophy of the calves. Regarding her upper limbs, there was 20 degrees of bilateral elbow flexion contracture and weakness of the small muscles of the hands bilaterally. Neurological examination revealed generalized hypotonia, nystagmus and diminished deep tendon reflexes. IQ evaluation showed moderate mental retardation. 
Table I: Genetic defects in various syndromic congenital muscular dystrophies.

\begin{tabular}{|llll|}
\hline Syndromic CMD* & Gene Symbol & Chromosomal Locus & Protein \\
\hline Fukuyama CMD* (FCMD) & FCMD & $9 \mathrm{q} 31$ & Fukutin \\
Muscle-eye-brain disease (MEB) & POMGNT1 & $1 \mathrm{p} 34-\mathrm{p33}$ & $\begin{array}{l}\text { ProteinO-mannosidebeta-1,2-N- } \\
\text { Walketylglucosaminyltransferase 1 }\end{array}$ \\
& & & $\begin{array}{l}\text { Protein O-mannosyl-transferase 1 } \\
\text { Walker-Warburg syndrome (WWS) }\end{array}$ \\
& POMT1 & $9 \mathrm{q} 34.1$ & Protein O-mannosyl-transferase 2 \\
\hline
\end{tabular}

*CMD- Congenital muscular dystrophy.

Table II: Differentiating features of various syndromic congenital muscular dystrophies.

\begin{tabular}{|llll|}
\hline Features & WWS* & MEBt & FCMD¥ \\
\hline Geographic distribution & Worldwide & Finland & Japan \\
Severity & Most severe & Moderate & Less severe \\
Typical ocular feature & Anterior chamber malformations, & High myopia, & cataract \\
& retinal dysplasia & cataract & \\
Contractures & Commonly elbow & Commonly elbow, knee & Elbow, hip, knee, ankle \\
Hydrocephalus & Common & Uncommon & Uncommon \\
Dandy-Walker malformation & Common & Uncommon & Uncommon \\
Brainstem involvement & Common, Flat Pons & Uncommon & Uncommon \\
Cerebral cortex & Type II Lissencephaly & Type II Lissencephaly & Type II Lissencephaly \\
Cerebellar involvement & Hypoplasia cysts & Vermis hypoplasia & Uncommon \\
Hypotonia & Generalized & Generalized & Generalized \\
\hline
\end{tabular}

* WWS: Walker-Warburg Syndrome

† MEB: Muscle-eye-brain disease

¥FCMD: Fukuyama congenital muscular dystrophy
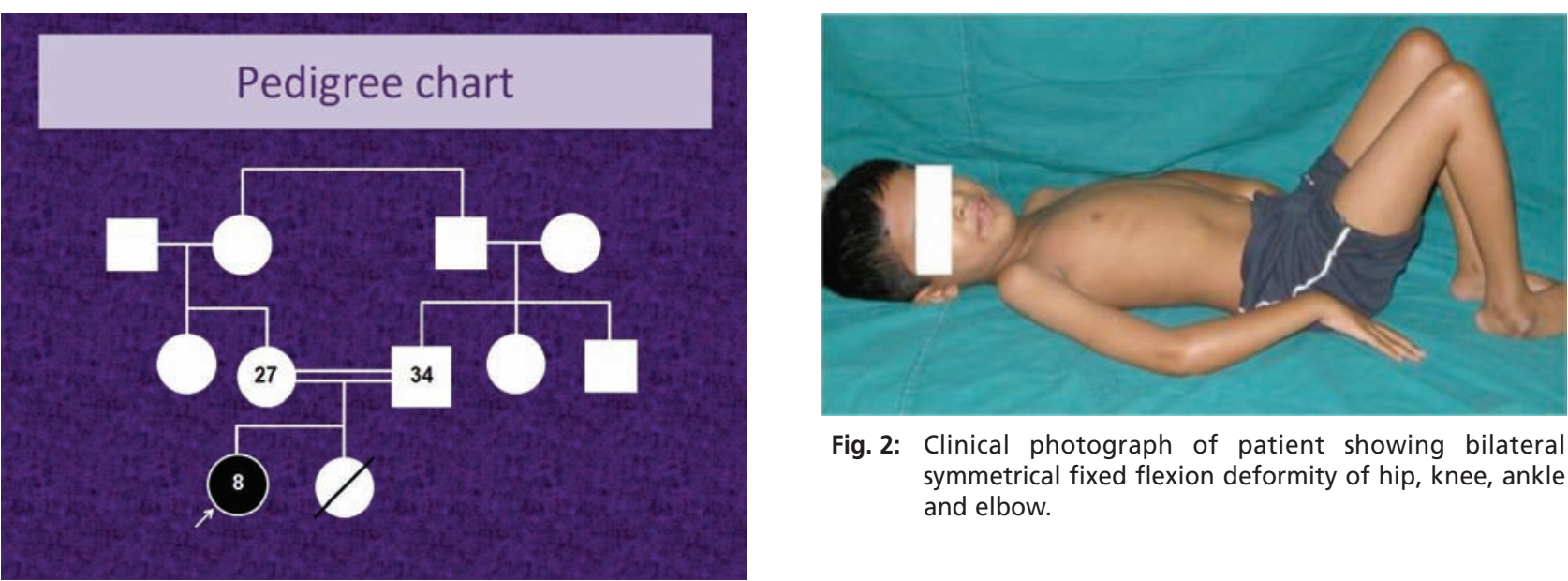

Fig. 2: Clinical photograph of patient showing bilateral symmetrical fixed flexion deformity of hip, knee, ankle and elbow.

Fig. 1: Pedigree chart showing first degree consanguinity.

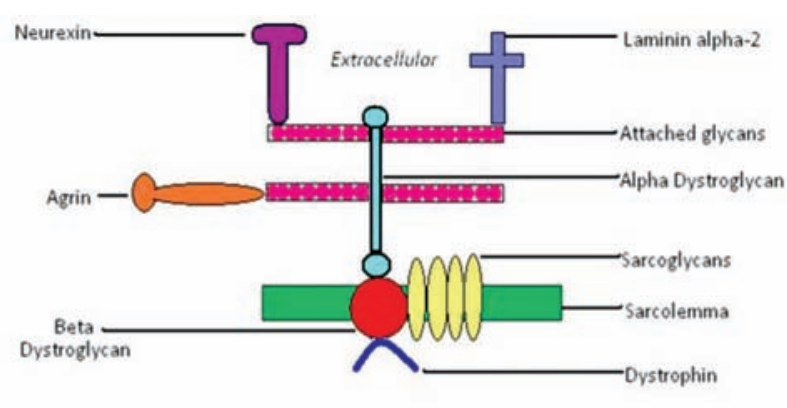

Introcellulor

Normal Muscle

Fig. 3a: Molecular biology of normal muscle.

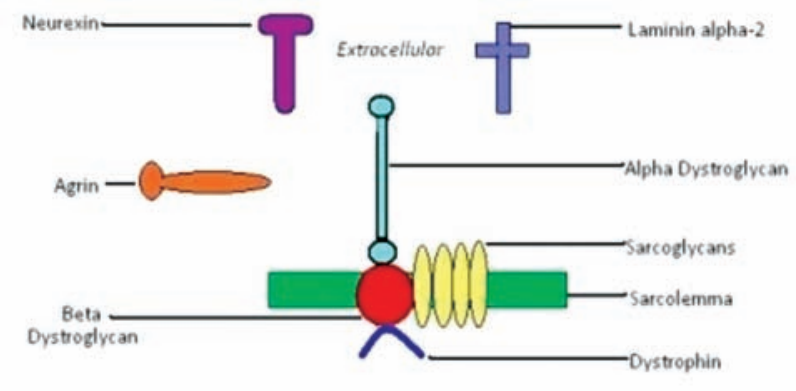

Introcelfular

\section{Dystrophic muscle}

Fig. 3b: Molecular biology of dystrophic muscle. 
Ophthalmology evaluation showed bilateral pseudophakia, a high degree of myopia with normal macula and optic disc. Serum creatine phosphokinase (CPK) was elevated at a level of $1150 \mathrm{u} / 1$ (normal range: 10-80 u / 1). MRI of the brain showed diffuse symmetrical T2 and FLAIR hyperintensities involving periventricular deep white matter of the frontoparietal lobes and pachygyria bilaterally with relative sparing of subcortical U-fibres and parieto-occipital and temporal lobes. There were tiny bilateral cerebellar cysts with hypoplasia of inferior vermis, all suggestive of type II lissencephaly. Muscle biopsy showed rounded myofibrils varying markedly in diameter, lateral nuclei, a few regenerating fibres and atrophic angulated fibres. There was a marked increase in fibrocollagenous tissue. Enzyme stains revealed type II fibre atrophy.

Based on the patient's history, clinical findings, elevated serum CPK, type II lissencephaly on brain MRI and dystrophic changes on muscle biopsy, a diagnosis of muscleeye-brain disease was made. The family was advised that she should undergo further molecular testing to identify the genetic defect which is necessary for prenatal diagnosis in future siblings; however, this was not accomplished due to financial constraints. Physicians explained to the family there is a $25 \%$ risk of recurrence in siblings.

\section{DISCUSSION}

Congenital muscular dystrophies (CMD) are a heterogeneous group of disorders characterized by congenital muscular hypotonia and the histologic features of muscular dystrophy. Based on the clinical, immunohistochemical, and genetic findings, different forms have been characterized. Syndromic CMDs include muscleeye-brain disease (MEB), Walker-Warburg syndrome(WWS) and Fukuyama congenital muscular
dystrophy(FCMD) all due to genetic defects in glycosyltransferases as mentioned in Table $\mathrm{I}^{1,2}$. There is failure of glycosylation of alpha-dystroglycans, which are transmembrane glycoproteins in the sarcolemma of skeletal muscles, eyes and brain tissue ${ }^{2,3,5}$. This leads to failure of ligand binding, intercellular interactions and hence dystrophic changes in these tissues (Figure $3 a$ and $3 b$ ). Though these syndromic congenital muscular dystrophies have overlapping clinical features, there remain certain subtle differences ${ }^{1,2}$, which help to distinguish among these various types of CMDs (Table II). T

Treatment is primarily supportive including: physical therapy and stretching exercises to promote mobility and prevent contractures or prevent them from worsening; use of mechanical assistive devices to help ambulation and mobility; and, correction of deformities based on the ambulatory capacity of the child. Genetic counselling should be offered to parents of such patients including avoidance of consanguineous marriage in the family since there is a twenty-five percent chance of each sibling of a proband to be affected. Utilization of prenatal testing in future siblings is also helpful.

No previous case report on muscle -eye-brain disease in India was found in the literature except for one case of another distinct variety of congenital muscular dystrophy (Fukuyama congenital muscular dystrophy) ${ }^{5}$. We speculate that muscle-eye-brain disease may be more common in India. It is imperative to consider this condition as a potential differential diagnosis when a child presents with persistent hypotonia or as a floppy baby. We suggest that registration of all neuromuscular disorders should be initiated in India so we may accurately calculate the prevalence of this condition and also work to prevent as well as rectify this crippling disorder in subsequent generations. 


\section{REFERENCES}

1. Reed UC. Congenital muscular dystrophy. Part I: a review of phenotypical and diagnostic aspects. Arq Neuropsiquiatr 2009; 67: 144-68.

2. Reed UC. Congenital muscular dystrophy. Part II: a review of pathogenesis and therapeutic perspectives. Arq Neuropsiquiatr 2009; 67: 343-62.

3. Mercuri E, Messina S, Bruno C, Mora M, Pegoraro E, Comi GP et al. Congenital muscular dystrophies with defective glycosylation of dystroglycans. Neurology 2009; 72: 1802-9.

4. Taniguchi K, Kobayashi K, Saito K, Yamanouchi H, Ohnuma A, Hayashi YK et al. Worldwide distribution and broader clinical spectrum of muscle-eye-brain disease. Hum Molec Genet 2003; 12: 527-34.

5. Garg A, Gulati S, Gupta V, Kalra V. Congenital muscular dystrophy with characteristic radiological findings similar to Fukuyama congenital muscular dystrophy. Neurol India 2004; 52: 496-8. 